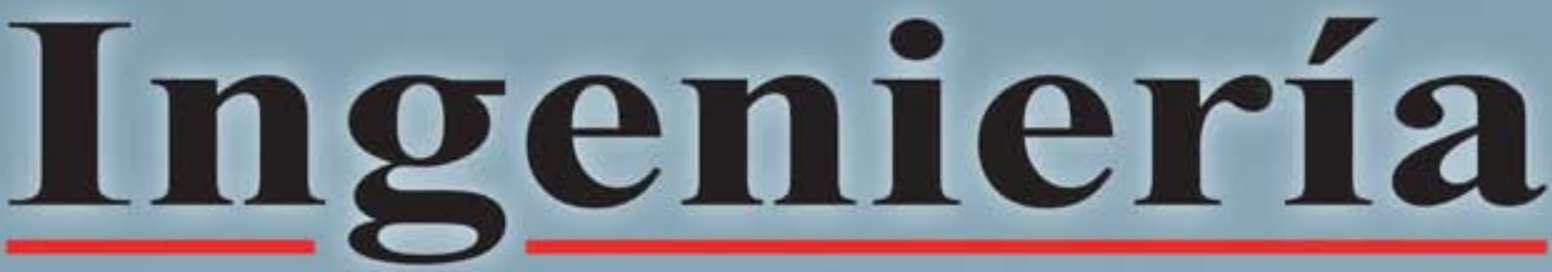

ENERO/DICIEMBRE 2004 - VOLUMEN 14 - N¹ y 2

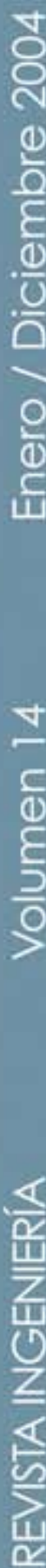
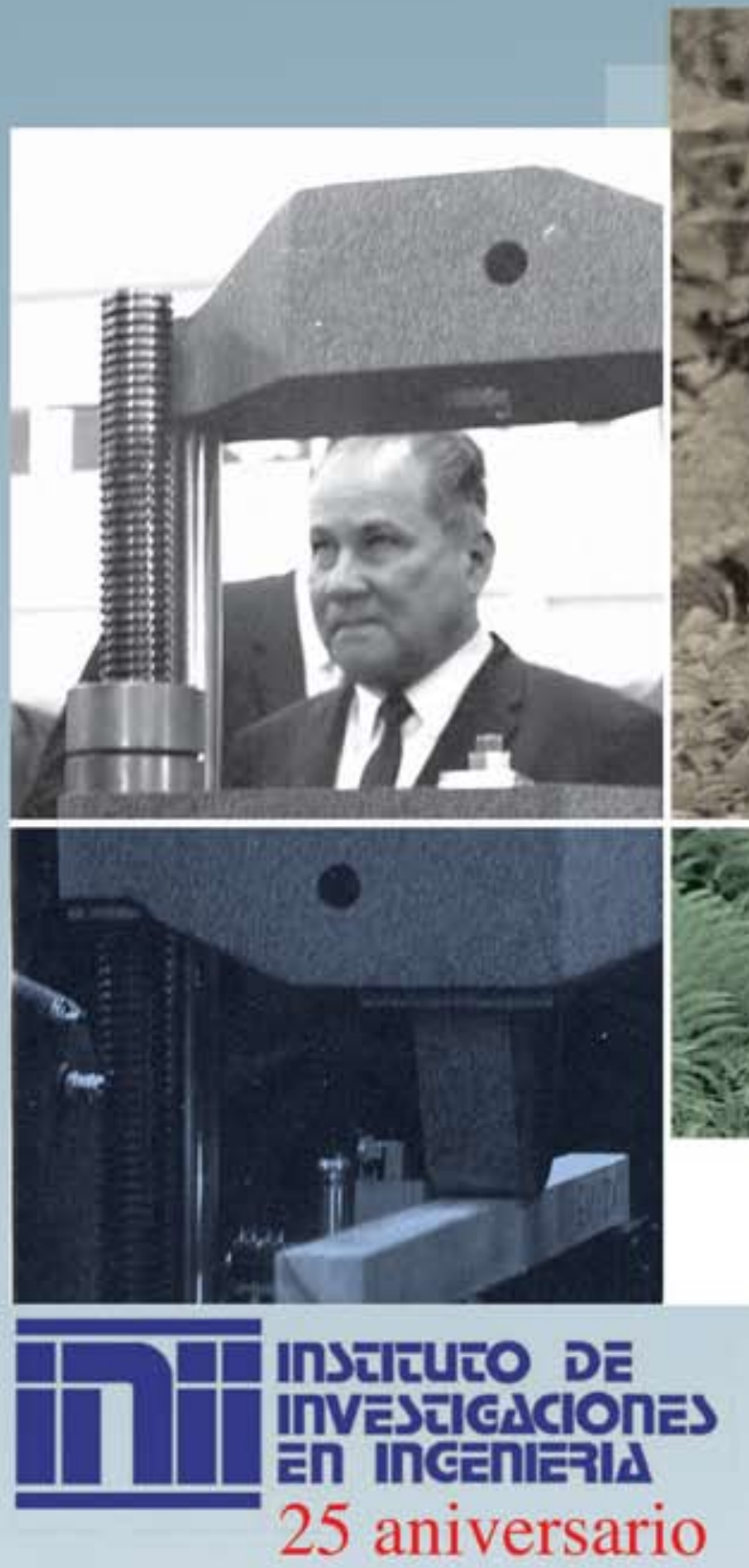

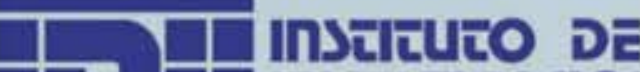
T W Investcscines हn Incenlei|d 25 aniversario

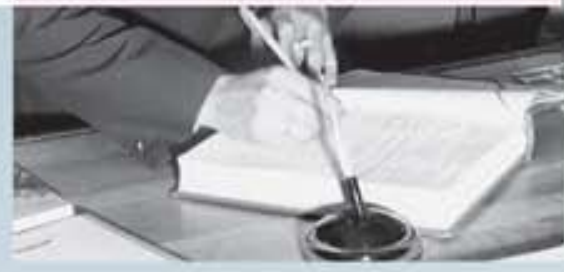




\title{
CÓDIGO SÍSMICO DE COSTA RICA VERSIÓN 2002. ESPECTROS DE RESISTENCIA PARA DUCTILIDAD CONSTANTE
}

\author{
Rubén Leandro Ulloa \\ Guillermo Santana Barboza
}

\begin{abstract}
Resumen
Este artículo es el segundo de una serie de tres investigaciones cuyo objetivo es validar las formas espectrales que se utilizan para el diseño sismorresistente de las estructuras civiles definidas en la nueva versión del Código Sísmico de Costa Rica (CSCR-2002) Con el uso de una base de datos de registros sísmicos, nacionales y extranjeros, se realizan 251596 corridas de sistemas de un grado de libertad para determinar los espectros de resistencia, en cada uno de los escenarios sísmicos obtenidos de la combinación de las zonas sísmicas y suelos de cimentación definidos por el CSCR-2002 en todos los valores de ductilidad. Los espectros de resistencia obtenidos con la base de datos de registros sísmicos presentan discrepancias, que en algunos, casos corresponde a subvaloraciones de los niveles de solicitación lateral que puedan actuar sobre la estructura.
\end{abstract}

Palabras clave: espectros de energía, ductilidad constante, espectros de diseño elástico, espectros de diseño inelástico, diseño sísmico, código de diseño sísmico, sistemas de un grado de libertad.

\begin{abstract}
This is the second part of a series of three investigations whose objective is to validate the spectral shapes that are used for the seismic-resistant design of the buildings defined into the new version of the Seismic Code of Costa Rica (CSCR-2002). With the use of a database of national and foreing seismic records, 251596 analysis of single degree of freedom systems were made to determinate the strength spectra according to each seismic scenary obtained from the combination of the seismic zones and foundation soils defined into the CSCR-2002 through the ductilities values. The strength spectra resulting from the seismic records database have discrepancies, which can correspond, in some cases, in lower values than the levels of the lateral forces than can be acting over the structure.
\end{abstract}

Keywords: strength spectra, constant ductility, elastic design spectra, inelastic design spectra, seismic design, seismic design code, single degree of freedom.

Recibido 08-VII-04 • Aceptado 14-X-04

\section{INTRODUCCIÓN}

Los eventos sísmicos ocurridos durante las últimas dos décadas en Costa Rica (sismo de Piedras Negras, sismo de Cóbano, sismo de Limón), a pesar de haber sido catalogados como sismos destructivos, por las magnitudes que alcanzaron y los daños que provocaron a la infraestructura, presentan valores bajos en los parámetros que definen el potencial de daño de un terremoto. Estudios llevados a cabo por investigadores nacionales (Santana, 1994 y Cruz, 1998) corroboran este hecho, al comparar los valores de los parámetros energéticos con los correspondientes de sismos extranjeros. Sin embargo, en algunos casos, para períodos estructurales pequeños, la demanda sísmica sobrepasó los requerimientos definidos por los espectros de diseño del Código Sísmico de Costa Rica vigente, versión 1986 (CSCR-1986) (Jiménez, 1996).

Esta situación propicia la necesidad de la realización de análisis más profundos, cuyo objetivo sea proponer formas espectrales, a partir de nueva información de registros de 
aceleraciones representativos, y compararlos con los espectros del CSCR-2002, logrando así validar los criterios de diseño existentes o modificarlos a los niveles requeridos.

\section{MODELO ESTRUCTURAL}

Consiste en un sistema de un grado de libertad, representado por un marco estructural formado por dos columnas con masa despreciable de idéntica sección y rigidez (con rigidez infinita en el sentido vertical y flexibles en el sentido horizontal, susceptibles a deformaciones laterales) las cuales se encuentran unidas en su parte superior por medio de un piso infinitamente rígido, en el cual se encuentra la masa distribuida de forma uniforme a lo largo de su longitud. Adicionalmente, para tomar en cuenta la disipación de energía, se cuenta con un amortiguador viscoso.

El modelo de comportamiento de material que se va a adoptar en el análisis es el bilineal, considerado como un caso general de buen nivel de simplicidad numérica, del cual son situaciones puntuales el modelo elástico y el modelo elástico perfectamente plástico. El valor de la rigidez secundaria o rigidez post fluencia, para efectos de este trabajo, será de $10 \%$ del valor de la rigidez inicial o rigidez elástica, valor considerado como estándar para análisis de espectros de diseño.

La ecuación de movimiento para un sistema de un grado de libertad que incursiona inelásticamente, es expresada como función de la ductilidad y de la razón adimensional de resistencia, y es la ecuación que resuelve el programa de análisis no lineal dinámico NONSPEC.

El programa NONSPEC fue diseñado por Mahin y Lin 1983 para analizar la respuesta inelástica de sistemas de un grado de libertad con amortiguamiento viscoso, sometidos a excitaciones oscilatorias o cargas directas externas. Está en capacidad de simular la respuesta para dos tipos de modelos de comportamiento, el bilineal y el de degradación de rigidez. El lenguaje de programación es FORTRAN 77. La ecuación de movimiento es resuelta por medio de métodos numéricos, debido a la inexistencia de un método analítico exacto. El NONSPEC emplea el método paso a paso, para obtener la respuesta.

La versión utilizada en este trabajo fue modificada por Santana (1995) para trabajar en ambiente Windows. Dicha versión fue adaptada para efectos de aumentar su capacidad de almacenamiento en cuanto a combinaciones de análisis y datos del registro de aceleraciones.

\section{CÓdIgo SÍSMICO DE COSTA RICA VERSIÓN 2002}

Se hace uso de la aceleración pico efectiva (EPA en inglés), la cual se convierte en un factor de normalización para la construcción de espectros de respuesta elásticos suavizados para sismos de duración normal. El EPA es proporcional a las ordenadas espectrales elásticas con un amortiguamiento del $5 \%$ para períodos en el rango de $0,1 \mathrm{~s}$ a $0,5 \mathrm{~s}$, con una constante de proporcionalidad de 2,5 .

La existencia del EPA conduce a la construcción de la zonificación sísmica, para un período de retorno de 475 años, en los cuáles se determina el potencial de EPA. El primer nivel de sismicidad corresponde hasta $0,2 \mathrm{~g}$, el segundo nivel hasta $0,3 \mathrm{~g}$ y el último para sismos con valores de EPA mayores a $0,4 \mathrm{~g}$.

Están definidos cuatro tipos de suelo de cimentación los cuales contemplan el horizonte desde perfiles rocosos (caracterizados por altas velocidades de propagación de la onda de cortante) hasta perfiles formados por estratos de suelo no cohesivos en los cuales ocurre amplificación de la señal sísmica.

Tipo $\mathbf{S}_{1}$ (Roca): Rocas volcánicas efusivas (basaltos y andesitas), intrusivas (granodioritas, monzonitas, gabros y sienitas), metamórficas 
(cornubianitas), poco fisuradas, con una velocidad de propagación de la onda cortante en el rango de $2500 \mathrm{~m} / \mathrm{s}$ a $4000 \mathrm{~m} / \mathrm{s}$.

Tipo $\mathbf{S}_{\mathbf{2}}$ (Firme): Rocas sedimentarias (areniscas, lutitas, calizas, brechas y conglomerados), rocas volcánicas (flujos piroclásticos, lavas y lahares), con espesores de arcilla inferiores a $20 \mathrm{~m}$ y velocidades de propagación de la onda cortante en el rango de $1500 \mathrm{~m} / \mathrm{s}$ a $1700 \mathrm{~m} / \mathrm{s}$.

Tipo $\mathbf{S}_{\mathbf{3}}$ (Blando): Rocas volcánicas (lavas y depósitos piroclastos), fuertemente alteradas, donde los niveles freáticos son altos, los espesores de arcilla de hasta $10 \mathrm{~m}$ sobre suelo tipo S2 y velocidad de propagación de la onda cortante en el rango de $800 \mathrm{~m} / \mathrm{s}$ a $1200 \mathrm{~m} / \mathrm{s}$.

Tipo $\mathbf{S}_{\mathbf{4}}$ (Muy blando): Rocas volcánicas (lahares, cenizas y tobas), rocas sedimentarias (diatomitas, lacustres y lutitas), depósitos recientes (aluviones y coluvios), de baja calidad mecánica, con niveles freáticos someros, son rocas fuertemente alteradas, espesores de arcilla mayores a $50 \mathrm{~m}$ sobre suelos tipo $\mathrm{S}_{1}, \mathrm{~S}_{2}$ y $\mathrm{S}_{3}$, velocidad de propagación de la onda cortante en el rango de $235 \mathrm{~m} / \mathrm{s}$ a $300 \mathrm{~m} / \mathrm{s}$.

Como complemento a los diferentes perfiles de cimentación, han sido definidas las zonas de incidencia sísmica de acuerdo con los niveles esperados de EPA. La zonificación sísmica de Costa Rica se muestra en la Figura 1.

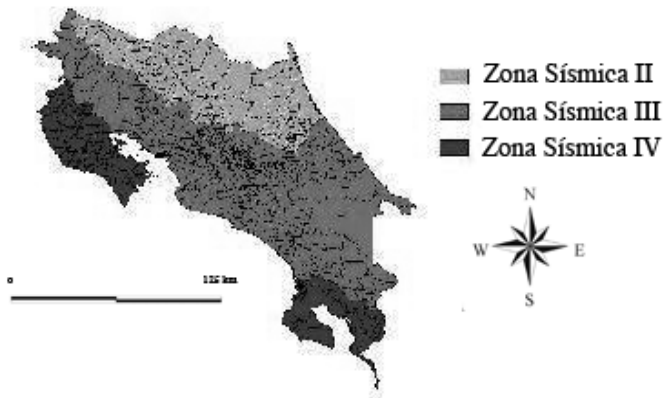

Figura 1. Mapa de zonas sísmicas.
La combinación de las tres zonas sísmicas $(\mathrm{Z} 2$, $\mathrm{Z} 3, \mathrm{Z} 4)$ y los cuatro suelos de cimentación $\left(\mathrm{S}_{1}\right.$, $\mathrm{S}_{2}, \mathrm{~S}_{3}, \mathrm{~S}_{4}$ ) conduce a la consideración de doce posibles escenarios en los cuales se deben definir los valores de las solicitaciones sísmicas que han de actuar sobre las estructuras construidas en dichos suelos y bajo esos niveles de potencial sísmico.

Con la determinación del EPA esperado, según la zona geográfica se hace uso de los espectros de diseño del Código (para un único valor de amortiguamiento del $5 \%$ ) en los cuales se presenta la relación con respecto al período de las formas espectrales dinámicas (FED) que al ser multiplicadas por el correspondiente valor de EPA conduce a los niveles de fuerza lateral (F) que han de actuar sobre el peso de la estructura (W) constituyendo así el procedimiento requerido para proceder al análisis sísmico de las estructuras.

$$
\begin{aligned}
& \mathrm{C}_{\text {Sís }}=\mathrm{FED} \bullet \mathrm{EPA} / \mathrm{g} \\
& \mathrm{F}=\mathrm{C}_{\text {Sís }} \cdot \mathrm{W}
\end{aligned}
$$

Se define la razón adimensional de resistencias de la siguiente forma:

$\eta=F E D\left(\frac{E P A}{A_{\max }}\right)$

el cual se encuentra relacionado con el coeficiente sísmico. Se aprovecha la siguiente ecuación que permite expresar en términos del coeficiente sísmico, tanto los factores adimensionales de resistencia como las formas espectrales dinámicas.

$$
\begin{aligned}
C_{s i s} & =\eta\left(\frac{A_{\max }}{g}\right) \\
C_{s i s} & =F E D \cdot(E P A)
\end{aligned}
$$

Para cada una de las doce combinaciones de suelo y zona sísmica, el CSCR-2002 define un conjunto de 6 espectros de diseño, uno de ellos elástico (ductilidad $=1$ ) y los cinco restantes inelásticos (ductilidades $=1,5,2,3,4,6$ ) para 
comprender las diversas tipologías estructurales que se puedan presentar (sistemas marcos, sistemas muros y sistemas duales).

\section{BASE DE DATOS DE REGISTROS SÍSMICOS}

Se hace uso de una base de datos de 36 registros sísmicos. Tres registros por cada combinación de zona sísmica $(\mathrm{Z} 2, \mathrm{Z} 3, \mathrm{Z} 4)$ con tipo de suelo $\left(\mathrm{S}_{1}\right.$, $\mathrm{S}_{2}, \mathrm{~S}_{3}, \mathrm{~S}_{4}$ ). La mayoría de los registros sísmicos están medidos en campo libre.

En el Cuadro 1 se encuentran representados veintidós diferentes eventos sísmicos (tres costarricenses, diez americanos, dos asiáticos, siete europeos) con magnitudes $M_{S}$ que se encuentran dentro del rango de 5,4 hasta 8,5 y magnitudes $M_{w}$ que se encuentran dentro del rango de 6,7 a 7,6. Las profundidades focales varían desde $5 \mathrm{~km}$ hasta $30 \mathrm{~km}$, indicando sismos de baja a mediana profundidad. Los mecanismos de falla consideran todos los tipos, con una presencia de doce eventos de falla inversa, nueve eventos de falla lateral y un evento de falla normal.

El Cuadro 2 presenta la información de los parámetros sísmicos para cada uno de los registros de la base de datos en el siguiente orden: tipo de suelo de cimentación, valor de aceleración máxima del terreno (PGA en inglés), intensidad de Arias, Potencia de Housner, raíz media cuadrática de la aceleración, duración total del sismo, duración efectiva según Trifunac y Brady, período de mayor amplificación del espectro de Fourier, intensidad de cruces de cero, aceleración pico efectiva, zona sísmica según CSCR-2002, velocidad pico efectiva, intensidad de Sandí, intensidad espectral de Housner, potencial destructivo, factor de intensidad característico y período predominante del sismo.

Cuadro 1. Características de los eventos sísmicos de la base de datos.

\section{EVENTO SÍSMICO, PAÍS}

Piedras Negras, Costa Rica
Cóbano, Costa Rica
Limón, Costa Rica
Kern County, Estados Unidos
Imperial Valley, Estados Unidos
México D. F., México
Loma Prieta, Estados Unidos
San Fernando, Estados Unidos
Parkfield, Estados Unidos
Northridge, Estados Unidos
Hyogoken Nanbu, Japón
Chi-chi, Taiwán
Whittier Narrows, Estados Unidos
Cape Mendocino, Estados Unidos
Managua, Nicaragua
Tabas-e-golshan, Irán
Montenegro, Yugoslavia
Alkion, Grecia
Ardal, Irán
Erzincan, Turquía
Racha, Georgia
Gazli, Uzbekistán

FECHA

22 de diciembre de 1990
25 de marzo de 1990
22 de abril de 1991
07 de diciembre de 1952
19 de mayo de 1940
19 de setiembre de 1985
18 de octubre de 1989
09 de febrero de 1971
28 de junio de 1966
17 de enero de 1994
16 de enero de 1995
21 de setiembre de 1999
01 de octubre de 1987
25 de abril de 1992
23 de diciembre de 1972
16 de junio de 1978
15 de abril de 1979
24 de febrero de 1981
06 de abril de 1977
13 de marzo de 1992
03 de mayo de 1991
17 de mayo de 7196

\section{MAGNITUD}

$\mathrm{MS}=5,7$

$\mathrm{MS}=5,7$

$\mathrm{MS}=7,6$

$\mathrm{MS}=7,7$

$\mathrm{MS}=6,3$

$\mathrm{MS}=8,5$

$\mathrm{MS}=7,1$

$\mathrm{MS}=6,5$

$\mathrm{MS}=6,4$

$\mathrm{MS}=6,7$

$\mathrm{MS}=6,9$

$\mathrm{MS}=7,6$

$\mathrm{MS}=5,7$

$\mathrm{MS}=7,1$

$\mathrm{MS}=6,2$

$\mathrm{MS}=7,5$

$\mathrm{MS}=6,9$

$\mathrm{MS}=6,7$

$\mathrm{MS}=7,4$

$\mathrm{MS}=6,8$

$\mathrm{MS}=5,4$

$\mathrm{MS}=7,0$

\section{PROFUNDIDAD MECANISMO FOCAL (km) FOCAL}

6

16

10

16

5

18

13

10

18

10

15

9,5

10

5

5

12

17

10
Inverso

Lateral

Inverso

Lateral izquierdo

Lateral derecho

Lateral derecho Inverso

Lateral derecho Inverso

Lateral derecho Lateral Inverso Inverso

Lateral izquierdo Inverso Inverso Normal Oblicuo

Lateral derecho Inverso Inverso 
高 舟

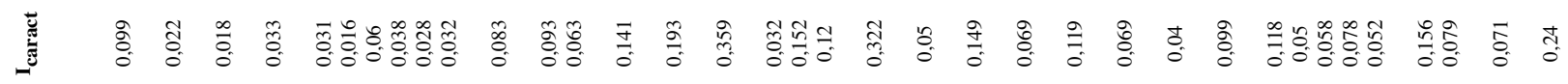

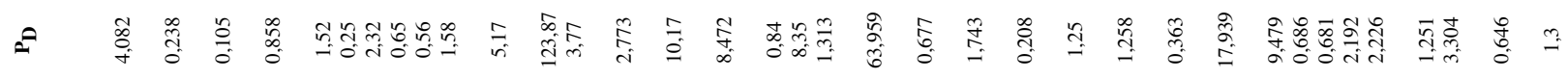
क 引 引

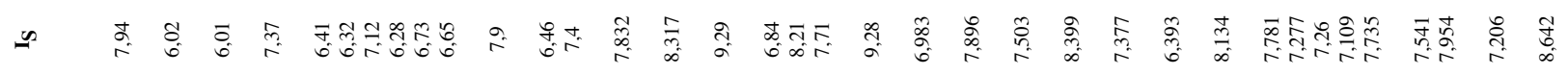

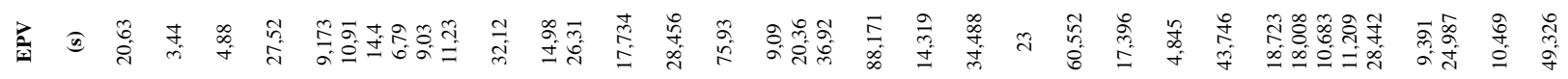

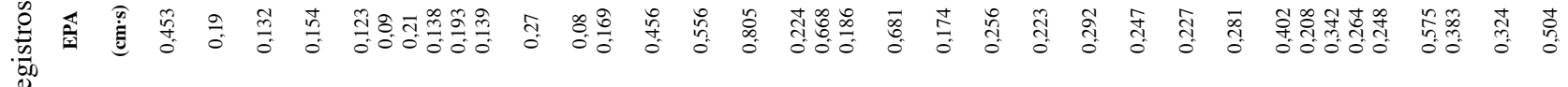

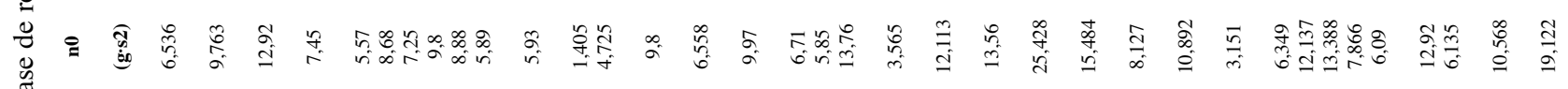

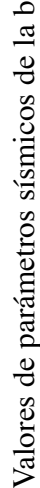

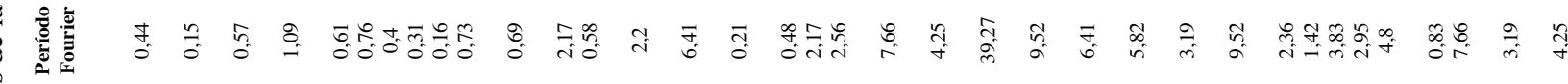
旁气

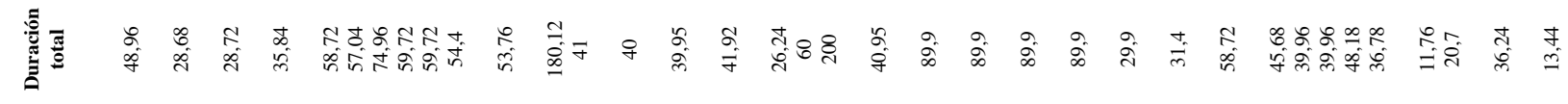

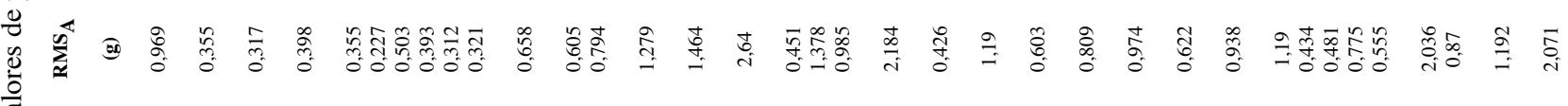

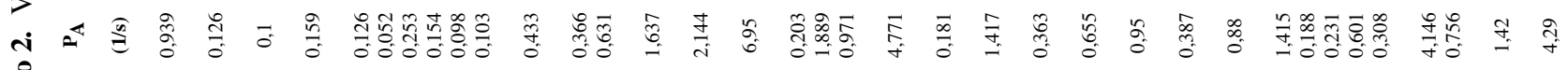
馬

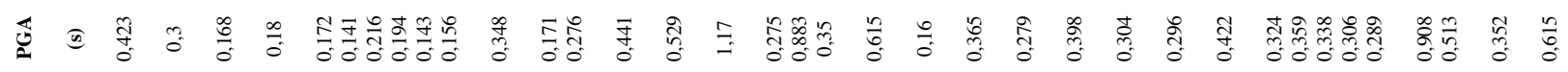

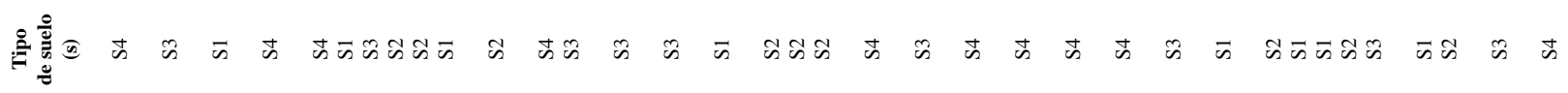

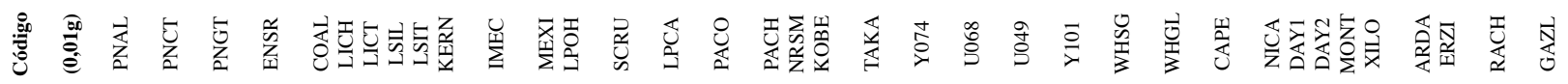

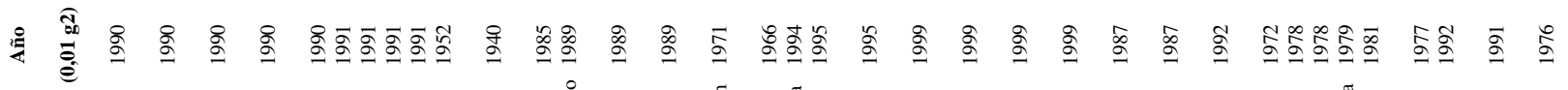

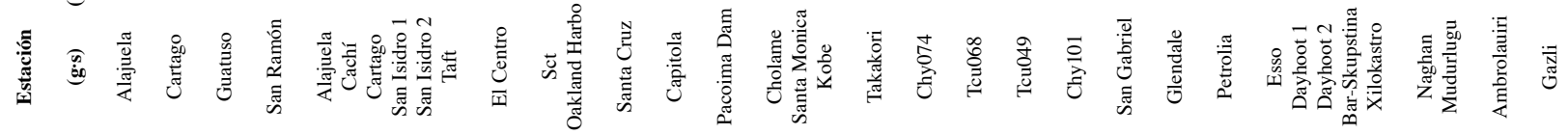

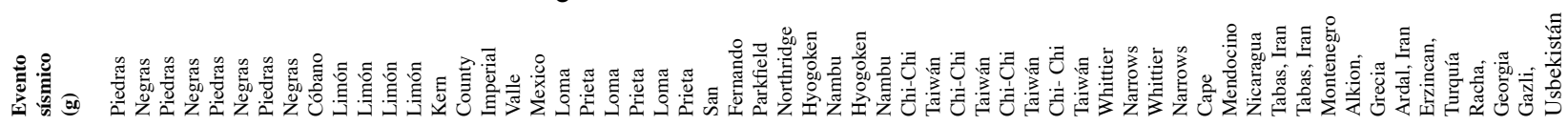


Del total de treinta y seis registros sísmicos de la base de datos, nueve son nacionales (25 $\%$ del total), los cuales corresponden a los eventos considerados de mayor potencial de daño registrados. Comparando estos eventos nacionales destructivos con los registros extranjeros (Cuadro 1), es posible determinar características de destructividad y daño potencial.

Los registros nacionales de la base de datos presentan promedios de duraciones efectivas (según criterio de Trifunac y Brady) mayores en un $23,84 \%$ al valor promedio de los registros extranjeros; sin embargo, esta situación no se debe interpretar a favor de un mayor grado de destructividad o potencial destructivo de los registros nacionales, ya que en los otros parámetros (aquellos relacionados con los valores picos del terreno, integraciones del registro en el tiempo, contenido de frecuencias, potenciales de daño) los registros extranjeros presentan, en conjunto, valores que son 54,67\% mayores a los registros nacionales. Lo anterior confirma lo indicado por investigadores nacionales Santana (1994) y Cruz (1998) acerca del potencial menor de daño que presentan los registros nacionales con respecto a los registros extranjeros, presumiendo la inexistencia de un registro nacional en el cual se obtenga un índice de destructividad mayor que los valores aquí indicados.

\section{COMPARACIÓN DE ESPECTROS DE RESISTENCIA ENTRE BASE DE DATOS Y CSCR-2002}

Para un rango de períodos estructurales comprendido entre $0,1 \mathrm{~s}$ y $4 \mathrm{~s}$, y con un amortiguamiento del $5 \%$ del amortiguamiento crítico, con el uso del programa NONSPEC se obtienen los espectros de resistencia. Para el caso de la base de datos, son utilizados los valores del promedio más la desviación estándar $(84,1$ percentil) los cuales se comparan con los valores medios obtenidos del CSCR-2002, y que tienen ya la consideración de la desviación estándar, debido al uso del criterio de Riddell y Newmark.
A partir de la Figura 2 y hasta la Figura 7, se presentan las relaciones gráficas de las comparaciones de los resultados obtenidos con el criterio de Miranda y los valores espectrales definidos en el CSCR-2002. Esto permite encontrar zonas en las cuales existan discrepancias numéricas entre estos valores.

Considerando que el rango de períodos cortos corresponde a valores comprendidos entre 0,1 s y $0,6 \mathrm{~s}$, que el rango de períodos intermedios corresponde a valores entre $0,6 \mathrm{~s}$ y $1,5 \mathrm{~s}$ y que los períodos largos son aquellos valores mayores a 1,5 s y redefiniendo al término "excedencia" como aquella situación en la cual los valores de las ordenadas espectrales obtenidas con el criterio de Miranda son mayores, a las leídas directamente de los espectros de diseño del CSCR-2002 obtenidas usando el criterio de Miranda, se observa lo siguiente:

Para el tipo de suelo $S_{1}$, conforme aumenta el valor del EPA, independiente de la ductilidad, los intervalos de excedencia aumentan, hasta llegar al caso de la zona sísmica Z4 en donde todo el rango de períodos excede al valor del CSCR2002. Se tiene como denominador común para las tres zonas sísmicas la presencia de excedencia en la zona de períodos intermedios relacionados con la región dominada por la velocidad.

Para el tipo de suelo $S_{2}$ y para todas las zonas sísmicas, independiente de la ductilidad, con excepción para la zona sísmica Z1 en los valores de ductilidades 3, 4 y 6, la zona de períodos cortos alrededor del valor de período de $0,2 \mathrm{~s}$ presenta excedencia. Para las zonas sísmicas Z3 y Z4, con excepción de la ductilidad 6 , existen intervalos de excedencia en los períodos largos rondando el valor de 2 s. En la zona sísmica Z3 para el rango de períodos intermedios en ductilidades 1 y 1,5 ocurre excedencia. Como denominador común en todas las zonas sísmicas, se presenta la excedencia en aquel sector espectral controlado por la aceleración. Además, ocurre excedencia en la zona controlada por el desplazamiento para las zonas sísmicas con valores altos de EPA, para las ductilidades menores a 6 . 
Para el tipo de suelo $\mathrm{S}_{3}$, en la zona sísmica Z2 ocurre excedencia en las tres regiones espectrales. Para la zona sísmica Z4 existe excedencia en el rango de períodos cortos para todas las ductilidades, y en la transición de los períodos intermedios a los períodos largos, esto en todas las ductilidades con excepción de ductilidad 6. La zona sísmica intermedia Z3 sólo presenta excedencia en los períodos cortos para ductilidades de 1 y 1,5. En las demás ductilidades de la zona sísmica Z3 no ocurre excedencia. La excedencia ocurre para todas las zonas sísmicas, independiente de la ductilidad, en la región controlada por la aceleración; sin embargo, en la zona sísmica Z3 para ductilidades mayores a 2, los valores calculados son similares a los del CSCR-2002.

Para el tipo de suelo $\mathrm{S}_{4}$, conforme aumenta el valor de EPA que caracteriza la zona sísmica, ocurre una reducción en el intervalo de períodos que no muestran excedencia. Independiente del valor de ductilidad, ocurre excedencia en las regiones controladas por el desplazamiento y por la aceleración en todas las zonas sísmicas. En la zona sísmica Z2 no se presenta excedencia para la región de velocidades en ductilidades menores de 2 .

Para la zona sísmica Z2 existe excedencia para los tipos de suelos $\mathrm{S}_{3}$ y $\mathrm{S}_{4}$ en todos los valores de ductilidad, en la región de períodos altos y ocurre excedencia en la región de períodos intermedios para ductilidades mayores a 3 .

Para la zona sísmica Z3 ocurre excedencia en valores de ductilidades menores a 2 y para todo tipo de suelo, con excepción de $\mathrm{S}_{3}$, ocurre excedencia en la zona de transición de períodos cortos a períodos intermedios. El tipo de suelo $\mathrm{S}_{3}$ presenta un comportamiento diferente al de los otros tipos de suelos, para ductilidades mayores a 2. Mientras los tipos de suelo $S_{2}$ y $S_{4}$ presentan excedencia para todas las ductilidades en la región de aceleraciones.

Para la zona sísmica Z4, de forma independiente al tipo de suelo y a la ductilidad, existe excedencia en la zona de períodos cortos. Solamente el tipo de suelo $S_{3}$, de forma independiente de la ductilidad, no presenta excedencia para períodos largos. Además, los tipos de suelo $\mathrm{S}_{1}$ y $\mathrm{S}_{4}$ presentan excedencia, independiente de los valores de ductilidad en la región la zona de velocidades.

\section{CONCLUSIONES}

1. Los registros nacionales más fuertes (Piedras Negras, Limón y Cóbano) no pueden ser considerados, en términos absolutos, sismos destructivos. Los parámetros sísmicos indican valores promedios que resultan muy pequeños, comparados con los valores de los registros extranjeros.

2. El CSCR-2002 no indica de manera directa los sismos que han sido considerados para la determinación de los espectros de diseño, ni el modelo de comportamiento estructural considerado. Dicha información es necesaria para realizar calibraciones de los espectros de resistencia inelásticas para una base de datos que sea más representativa de la sismicidad de las regiones geográficas definidas por este código.

3. El coeficiente sísmico obtenido con la base de registros sísmicos para cada una de las combinaciones de zona sísmica y suelo de cimentación, presenta valores mayores a los obtenidos directamente de los espectros de diseño del CSCR-2002 en un rango importante de períodos, el cual se va haciendo más amplio conforme se pasa a los perfiles de suelo que tienen más posibilidad de amplificación de la respuesta sísmica (perfiles menos rígidos del suelo).

4. La obtención de las fuerzas sísmicas a partir de los espectros de diseño para la posterior selección y detallado de las secciones estructurales de las obras civiles, puede conducir a subvalorar la demanda sísmica y realizar diseños poco conservadores, de acuerdo con los resultados de la base de registros sísmicos. 


\section{SIMBOLOGÍA}

\begin{tabular}{|c|c|}
\hline$v_{0}=\frac{\# z . c .}{t_{B}}$ & $\begin{array}{l}\text { Intensidad de cruces } \\
\text { de cero (Saragoni), } \mathrm{s}^{-1}\end{array}$ \\
\hline $\mathrm{A}_{\max }$ & $\begin{array}{l}\text { Aceleración máxima } \\
\text { del terreno }\end{array}$ \\
\hline $\mathrm{C}_{\text {sis }}$ & $\begin{array}{l}\text { Coeficiente sísmico, } \\
\text { (adimensional) g }\end{array}$ \\
\hline EPA & $\begin{array}{l}\text { Aceleración pico efectiva } \\
\text { de acuerdo al ATC, g }\end{array}$ \\
\hline EPV & $\begin{array}{l}\text { Velocidad pico efectiva de } \\
\text { acuerdo al ATC, } \mathrm{cm} / \mathrm{s}\end{array}$ \\
\hline $\mathrm{I}_{\mathrm{A}}=\frac{\pi}{2 g} \int_{0}^{t_{d}} \ddot{u}_{g}^{2}(t) d t$ & Intensidad de Arias, $(g \bullet s)$ \\
\hline $\mathrm{I}_{\text {caract }}$ & $\begin{array}{l}\text { Intensidad característica, } \\
\mathrm{g}^{1,5} \cdot \mathrm{s} 0,5\end{array}$ \\
\hline $\mathrm{I}_{\mathrm{S}}=\log _{4}(E P A \cdot E P V)+I_{0}$ & $\begin{array}{l}\text { Intensidad de Sandi } \\
\text { con } \boldsymbol{I}_{0}=8,0\end{array}$ \\
\hline $\mathrm{P}_{\mathrm{A}}=1 \int^{t_{0.95}}\left(\ddot{i}_{0}^{2}(t) d t\right.$ & Potencia sísmica de \\
\hline$t_{0,95} \quad t_{0,05} \int_{t_{0,05}}$ & \\
\hline $\mathrm{P}_{\mathrm{D}}=\frac{I_{A}}{v_{o}^{2}}$ & $\begin{array}{l}\text { Coeficiente de destructividad } \\
\text { (Saragoni), } \mathrm{cm} \bullet \mathrm{s}\end{array}$ \\
\hline PGA & $\begin{array}{l}\text { Aceleración pico del } \\
\text { terreno, } \mathrm{g}\end{array}$ \\
\hline $\mathrm{RMS}_{\mathrm{A}}=\sqrt{P_{A}}$ & $\begin{array}{l}\text { Raíz media cuadrática, } \\
10^{-2} \mathrm{~g}\end{array}$ \\
\hline $\mathrm{SI}(\boldsymbol{\xi}=\mathbf{5} \%)=\int_{0,1}^{2.5} S_{p v}(\xi, T) d T$ & $\begin{array}{l}\text { Intensidad espectral de } \\
\text { Housner, } g \bullet s^{2}\end{array}$ \\
\hline$t_{B}$ & $\begin{array}{l}\text { Duración enmarcada } \\
(>0,05 \mathrm{~g}) \text { (Bolt), } \mathrm{s}\end{array}$ \\
\hline$t_{D}=t_{0,95}-t_{0,05}$ & $\begin{array}{l}\text { Duración } \\
\text { (Trifunac \& Brady), s }\end{array}$ \\
\hline Tsism & $\begin{array}{l}\text { Periodo predominate } \\
\text { del sísmo, s }\end{array}$ \\
\hline
\end{tabular}

\section{REFERENCIAS BIBLIOGRÁFICAS}

Chopra, A. (1995). Dynamics of structures: theory and applications to earthquake engineering. New Jersey, N. Y. : Prentice Hall.

Colegio Federado de Ingenieros y Arquitectos, (2002). Gutiérrez, J. (editor). Código Sísmico de Costa Rica. Versión 2002. Cartago: Editorial Tecnológica de Costa Rica.

Cruz, M. \& et al. (1998). Evaluación del potencial de daño de sismos recientes en Costa Rica (pp.163-174). Memorias del X Seminario Latinoamericano de Ingeniería Sísmica.
Denyer, P. \& Kussmaul, S. (1994). Atlas geológico del Gran Área Metropolitana ( $1^{\mathrm{a}}$ ed.). Cartago, San José: Editorial Tecnológica de Costa Rica.

Fajfar, P. (1995). Elastic and inelastic design spectra. En: $10^{\text {th }}$ European Conference on Earthquake Engineering (pp. 1169-1178). Balkema, Rotterdam.

Fajfar, P. \& Vidic, T. (1994). Consistent inelastic design spectra: hysterestic and input energy. Earthquake Engineering and Structural Dynamics, 23, 523-537.

Fajfar, P., Vidic, T. \& Fischinger, M. (1989). Seismic demand in medium- and longperiod structures. Earthquake Engineering and Structural Dynamics, 18, 1133-1144.

Gupta, A. (1992). Response spectrum method in seismic analysis and design of structures, CRC Press. Florida, Estados Unidos de América.

Jiménez, O. (1996). Espectros inelásticos del terremoto de Limón de 1991. Trabajo de Graduación para Licenciatura en Ingeniería Civil, Escuela de Ingeniería, Universidad de Costa Rica, San José, Costa Rica.

Kawashima, K., Hoshikuma, J. \& Nagaya, K. (1998). Residual displacement response spectra. Journal of Structural Engineering, 124 (5), 523-530.

Lai, S. \& Biggs, J. (1980). Inelastic response spectra for aseismic building design. Journal of Structural Division ASCE, 106 (ST6), 1295-1310.

Mahin, S. \& Bertero, V. (1981). An evaluation of inelastic seismic design spectra. Journal of the Structural Division, 107 (9), 1777-1795.

Mahin, S. \& Lin, L. (1983). Construction of inelastic response spectra for single-degreeof-freedom systems. University of California. Earthquake Engineering Research Center

Miranda, E. (1995). Nonlinear response spectra for earthquake resistant design. En: $10^{\text {th }}$ European Conference on Earthquake 
Engineering (pp. 5835-5840). Balkema, Rotterdam.

Directorate of Licensing U.S. Atomic Energy Commission (1973). A study of vertical and horizontal earthquake spectra. (Report No. WASH-1255). Mohraz, B., Hall, W. \& Newmark, N.

Nassar, A., Osteraas, J. \& Krawinkler, H. (1995). Seismic design based on strength and ductility demands. En: $10^{\text {th }}$ European Conference on Earthquake Engineering (pp. 5861-5866). Balkema, Rotterdam.

Newmark, N. \& Hall, W. (1973). Seismic design criteria for nuclear reactor facilities. Building Practices for Disaster Mitigation, National Bureau of Standards (pp. 209-236). U.S. Department of Commerce.

Newmark, N. \& Hall, W. (1982). Earthquake Spectra and Design California, United States of America: Earthquake Engineering Research Institute.

Ochi, K. \& Kondo, H. (1995). Energy input rate spectra of earthquake ground motions. En: $10^{\text {th }}$ European Conference on Earthquake Engineering (pp. 641-644). Balkema, Rotterdam.

Ramírez, R., Santana, G., Chacón, O. (1995). Mapa de amplificación sísmica del Valle Central. San José, Costa Rica.

Riddell, R. \& Newmark, N. (1979). Statistical analysis of the response of nonlinear systems subjected to earthquakes Structural Research Series No. 48. Urbana, USA: University of Illinois, Department of Civil Engineering,

Riddell, R. (1989). Response modification factors for earthquake resistant design of short period buildings, Earthquake Spectra, 5 (3), 571-590.
Santana, G. \& Terán-Gilmore, A. (1994). Damage potential of earthquake ground motions recorded in southern Central America. Ingeniería, 4 (2), 75-88.

Santana, G. (2002) Nueva zonificación sísmica de Costa Rica. En: Seminario de Maestría, Escuela de Ingeniería Civil, Universidad de Costa Rica

Seed, H. \& Idriss, I. (1971). Influence of soil conditions on building damage potential during earthquakes. Journal of Structural Division, 97 (2), 639-663.

Veletsos, A. \& Newmark, N. (1960). Effects of inelastic behavior on the response of simple systems to earthquake ground motions. En: 2nd World Conference on Earthquake Engineering (pp. 895-912). Japan.

Vidic, T., Fajfar, P. \& Fischinger, M. (1994). Consistent inelastic design spectra: strength and displacement. Earthquake Engineering and Structural Dynamics, 23, 507-521.

\section{SOBRE LOS AUTORES}

\section{Sc. Rubén Leandro Ulloa.}

Magíster Scientaiae en Ingeniería Civil con énfasis en Estructuras, de la Universidad de Costa Rica, investigador independiente

Teléfonos: 392-7676 y 237-1570

Facsímil: 261-2382

Correos electrónicos:rlulloa@cfia.or.cr;

ruben.leandro@amanco.com

\section{Ph. D. Guillermo Santana Barboza.}

Ingeniero civil, profesor catedrático e investigador de la Escuela de Ingeniería Civil, de la Universidad de Costa Rica..

Teléfono: 207-4012

Facsímil: 207-4440

Correo electrónico: santana@lanamme.ucr.ac.cr 
a)

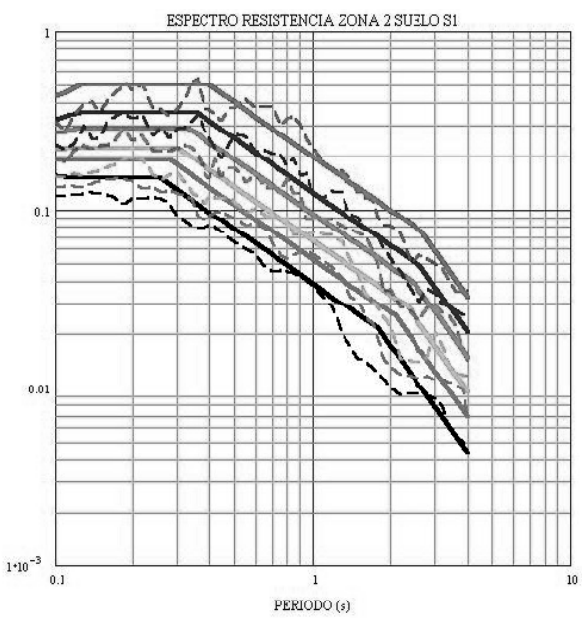

c)

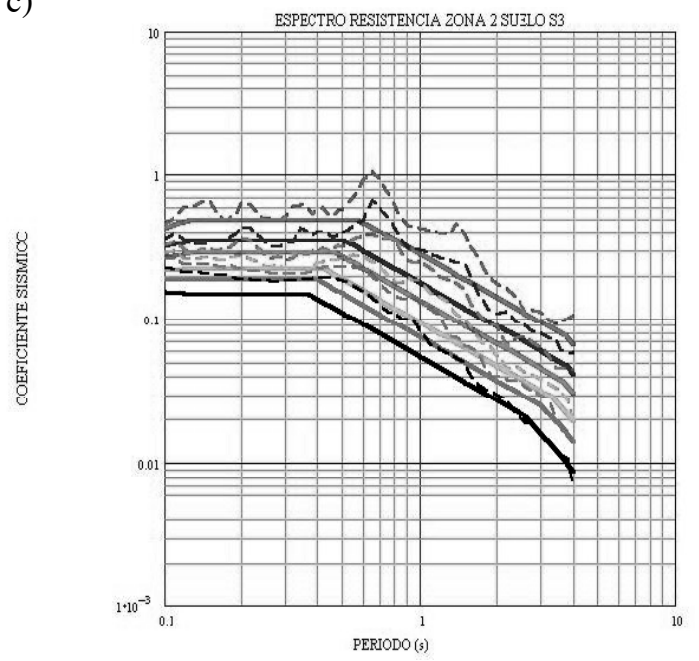

b)

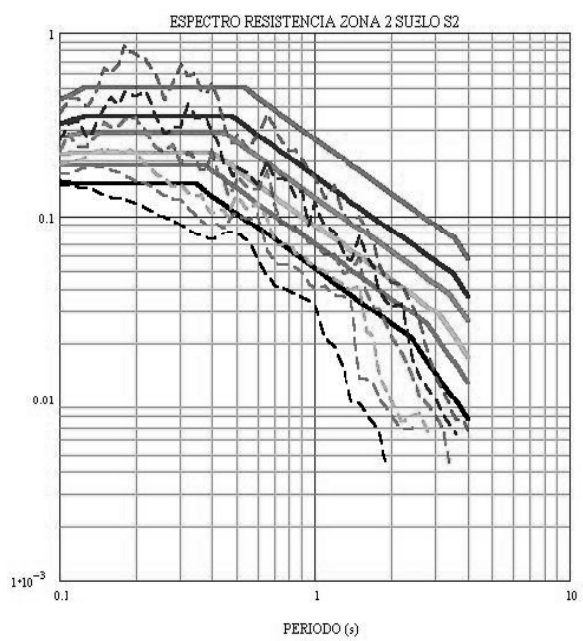

d)

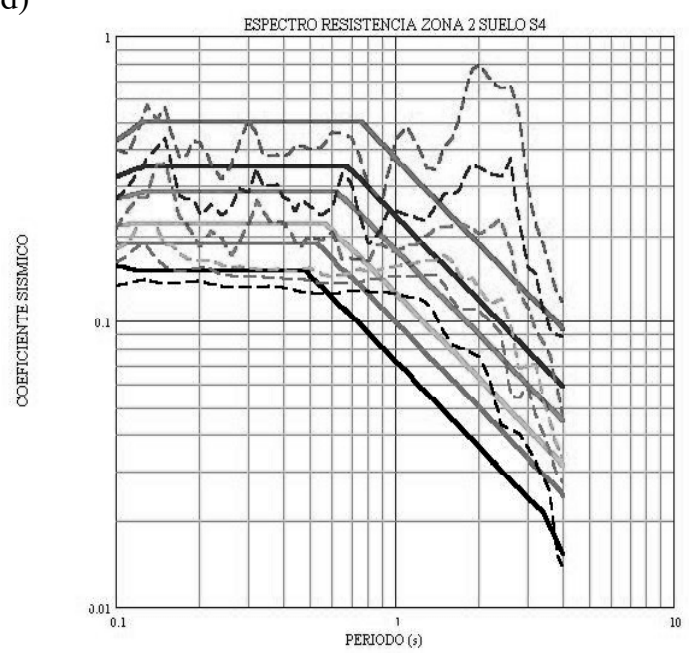

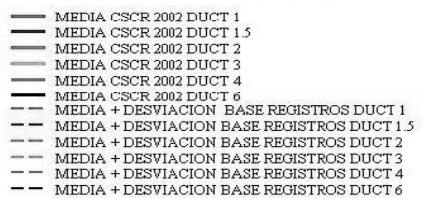

Figura 1. Comparación de espectros de diseños Zona sísmica y suelo cimientación (Base de registros sísmicos versus CSCR-2002): a) Z2 S1, b) Z2 S2, c) Z2 S3, d) Z2 S4

Ver imágenes a color en la página 167. 
a)

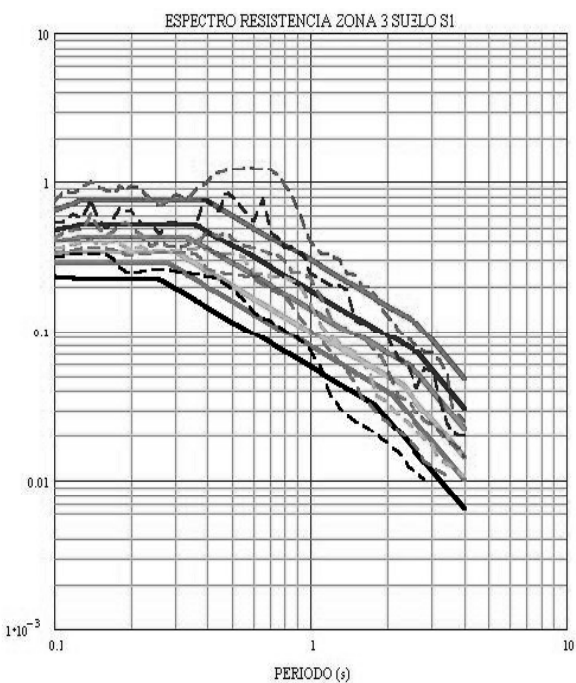

c)

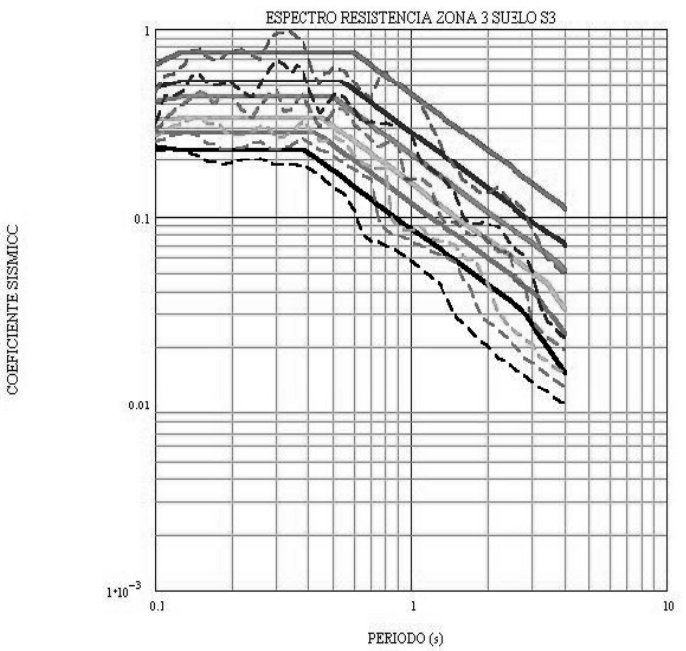

b)

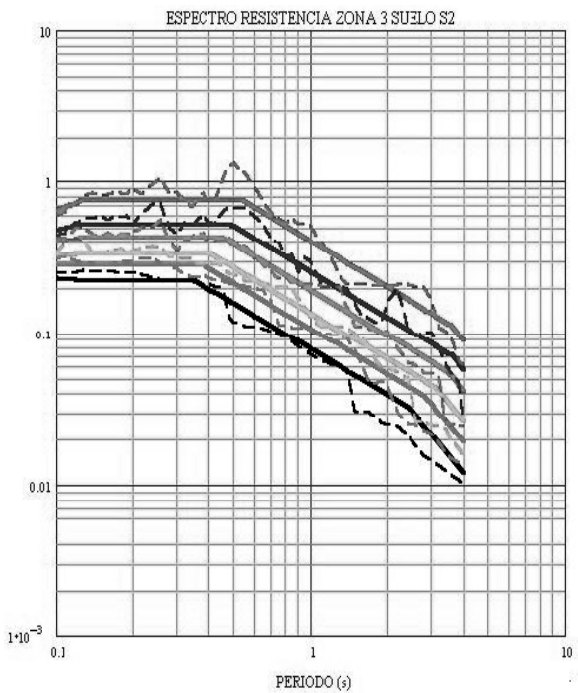

d)

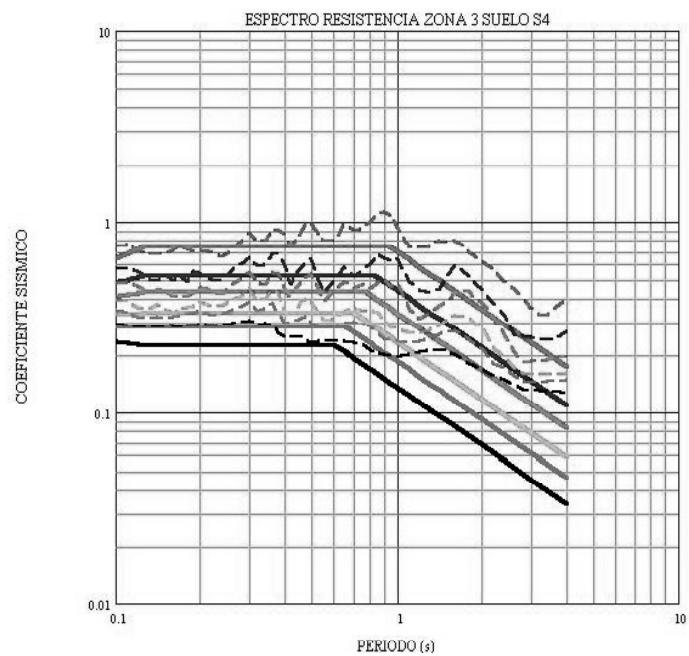

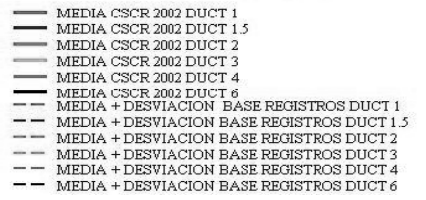

Figura 2. Comparación de espectros de diseños Zona sísmica y suelo cimientación (Base de registros sísmicos versus CSCR-2002): a) Z3 S1, b) Z3 S2, c) Z3 S3, d) Z3 S4

Ver imágenes a color en la página 168. 
a)

a)

c)

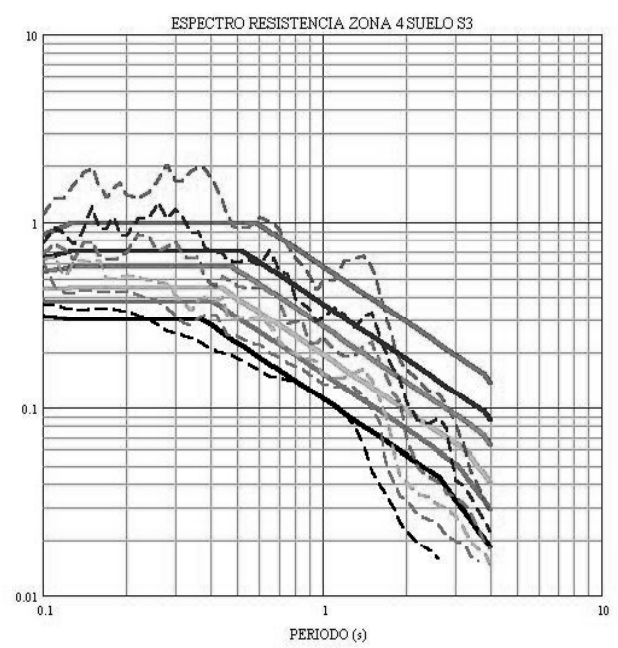

b)
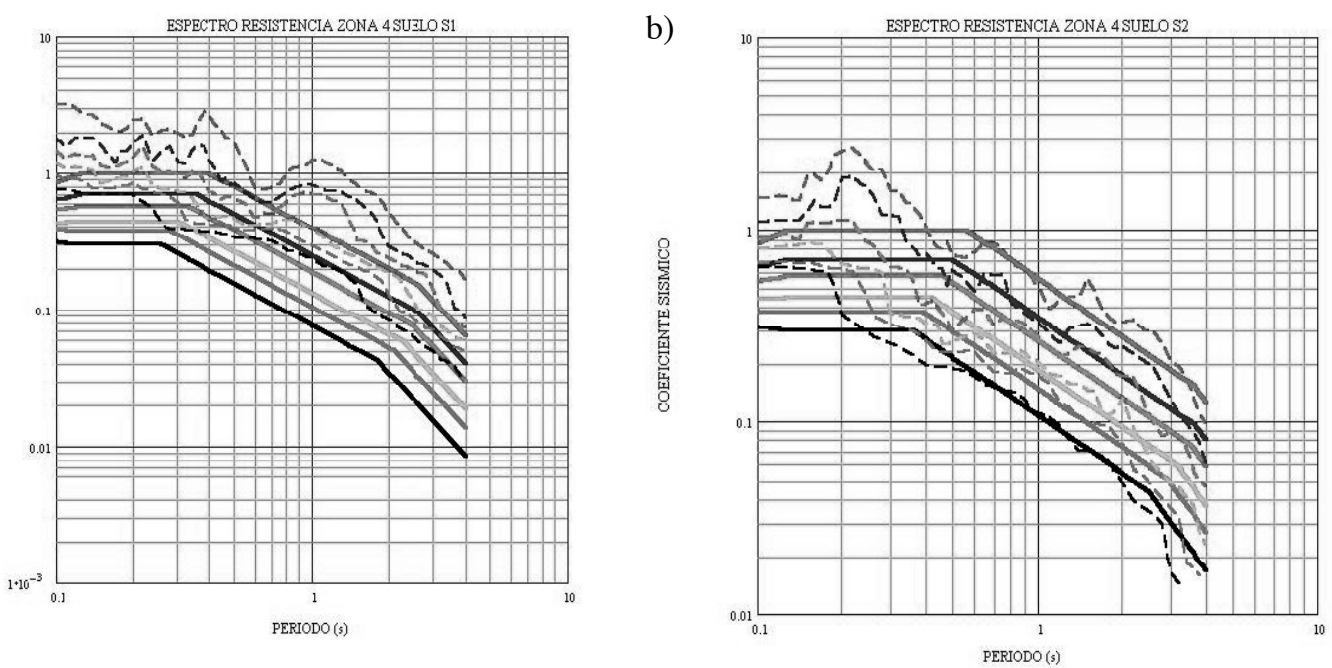

d)

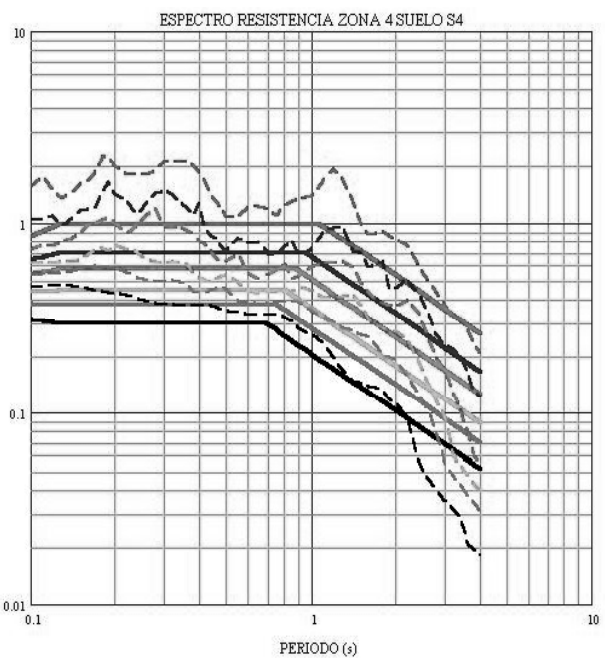

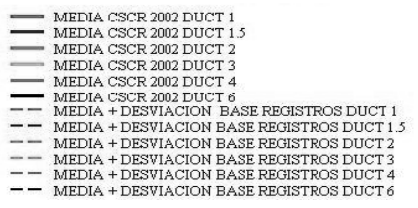

Figura 3. Comparación de espectros de diseños Zona sísmica y suelo cimientación (Base de registros sísmicos versus CSCR-2002): a) Z4 S1, b) Z4 S2, c) Z4 S3, d) Z4 S4

Ver imágenes a color en la página 169. 